\title{
A Micro Local Approach to General Boundary Value Problems
}

by

\author{
Kiyoomi KATAOKA*
}

\section{§ 0. Introduction}

Since the theory of non-characteristic boundary value problems in the category of hyperfunctions was initiated by H. Komatsu-T. Kawai, and P. Schapira independently ([8], [11]), this theory has made a remarkable progress. ([5], [6], [2]) But the methods do not seem to be sufficient for more general problems, for example, hyperbolic mixed problems.

Recently, a micro local formulation of non-characteristic boundary value problems was found by the auther ([7]), and it looks like very useful to understand and analyse general boundary value problems. So in this note we try to survey this formulation and the related results. Furthermore readers should refer to the results about mixed problems which were announced by P. Schapira at this symposium. ([12])

\section{$\S 1$. The Definitions of Two Sheaves and Their Properties}

Let $M$ be an $n$-dimentional real analytic manifold, $N$ be a submanifold of $M$ of codimention 1. For simplicity, from now on, we assume $M=\boldsymbol{R}^{n}$ $\exists\left(x_{1}, x^{\prime}\right), N=\left\{x \in M ; x_{1}=0\right\}$, and $M_{+}=\left\{x \in M ; x_{1} \geqq 0\right\}$. In the theory of boundary value problems, two sheaves, $\Gamma_{M^{+}} \mathscr{B}_{M}$ and $\Gamma_{N} \mathscr{B}_{M}$, play an important role. So it is necessary for the micro local formulation to analyse these sheaves micro locally. Then we introduce two sheaves, $\mathscr{C}_{M^{+} / X}, \mathscr{C}_{N / X}$. The latter has already been introduced and deeply studied by Kashiwara-Kawai in [4], [5], [6].

Received August 24, 1976.

* Department of Mathematics, Faculty of Sciences, University of Tokyo, Hongo, Bunkyoku, Tokyo, Japan. 
Let $X$ and $Y$ be complex neighborhoods of $M$ and $N$ respectively, then, $X=\mathbb{C}^{n} \ni\left(z_{1}, z^{\prime}\right), Y=\left\{z \in C^{n} ; z_{1}=0\right\}$,

$$
\begin{aligned}
& T^{*} X=\boldsymbol{C}^{n} \times \boldsymbol{C}^{n} \ni\left(z ; \zeta_{1}, \cdots, \zeta_{n}\right), \\
& T X=\boldsymbol{C}^{n} \times \boldsymbol{C}^{n} \ni\left(z ; \sum_{j=1}^{n}\left(w_{j} \frac{\partial}{\partial z_{j}}+\bar{w}_{j} \frac{\partial}{\partial \bar{z}_{j}}\right)\right) .
\end{aligned}
$$

Here, the inner product of $T X$ and $T^{*} X$ is defined by $-\operatorname{Re}\left(w_{1} \zeta_{1}+\cdots\right.$ $\left.+w_{n} \zeta_{n}\right)$, and $w=u+i v, \zeta=\xi+i \eta$.

Definition 1.1. We can define the conormal spherical bundle of $M_{+}$in $X$ as a closed subset of $S^{*} X$ in the following way:

$$
S_{M^{+}}^{*} X=\left\{(x ; i \eta) \in S_{M}{ }^{*} X ; x_{1} \geqq 0\right\} \cup\left\{\left(0, x^{\prime} ; \zeta_{1}, i \eta^{\prime}\right) \in S_{N} * X ; \operatorname{Re} \zeta_{1} \geqq 0\right\}
$$

Moreover we define; $G_{+}=\left\{\left(0, x^{\prime} ; \zeta_{1}, i \eta^{\prime}\right) \in S_{N}{ }^{*} X ; \operatorname{Re} \zeta_{1}>0\right\}$

$$
\begin{aligned}
& H_{+}=\left\{(x ; i \eta) \in S_{M}{ }^{*} X ; x_{1}>0\right\}, I=S_{M}{ }^{*} X \cap S_{N}{ }^{*} X, \\
& \pi_{M^{+} / X} ; S_{M_{+}}^{*} X \rightarrow M_{+} \text {(projection). }
\end{aligned}
$$

Then easily to see, these depend only on $M$ and $N$, and have intrinsic meanings. Especially,

$$
S_{M^{+}}^{*} X=G_{+} \cup I \cup H_{+}, \bar{G}_{+}=G_{+} \cup I, \bar{H}_{+}=H_{+} \cup I .
$$

Now we define the comonoidal transformation of $X$ with center $M_{+}$;

$$
\widetilde{M}_{X^{*}}^{+}=\left(X-M_{+}\right) \cup S_{M^{+}}^{*} X
$$

Then $\widetilde{M_{X^{*}}^{+}}$will be canonically equipped with a topological structure, and the projection mapping becomes continuous.

$$
\pi_{M^{+} / X} ; \widetilde{M_{X^{*}}^{+}} \rightarrow X \text { (cf. S-K-K, [10]). }
$$

Defimition 1.2. We denote by $\mathcal{O}_{X}$ the sheaf of holomorphic functions defined on $X$. Then by the theorem of the edge of the wedge, $S_{M^{+}}^{*} X\left(S_{N}{ }^{*} X\right)$ is purely $n$-codimentional for $\pi_{M^{+} / X}^{-1} \mathcal{O}_{X}\left(\pi_{N / X}^{-1} \mathcal{O}_{X}\right)$. (see, Morimoto [9], Kashiwara [3]) Therefore by the remaining cohomology groups we define;

$$
\mathscr{C}_{M_{+} / X}=\mathcal{H}_{S_{M+}^{*} X}^{n_{1}}\left(\pi_{M_{+} / X}^{-1} \mathcal{O}_{X}\right), \quad \mathscr{C}_{N / X}=\mathcal{H}_{S_{N}^{*} X}^{n^{*}}\left(\pi_{N / X}^{-1} \mathcal{O}_{X}\right)
$$

(here, we omit the orientation sheaves) 
Proposition 1.3. $\Gamma_{M^{+}} \mathscr{B}_{M} \stackrel{\sim}{\longrightarrow} \pi_{M_{+} / X^{*}} \mathscr{C}_{M_{+} / X}$, on $N$.

$$
O \rightarrow \Gamma_{N} \mathscr{B}_{M} \rightarrow \pi_{N / X^{+}} \mathscr{C}_{N / X} \rightarrow \mathcal{O}_{X} \rightarrow 0 \text {; exact on } N \text {. }
$$

Proposition 1.4. $\left.\quad \mathscr{C}_{M_{+} / X}\right|_{H_{+}}=\left.\mathscr{C}_{M}\right|_{H_{+}},\left.\mathscr{C}_{M_{+} / X}\right|_{G_{+}}=\left.\mathscr{C}_{N / X}\right|_{G^{+}}$

On I, the following sheaf homomorphisms are canonically induced, and these mappings are injective.

$$
\left.\left.\left.\mathscr{C}_{N / X}\right|_{I} \rightarrow \mathscr{C}_{M+/ X}\right|_{I} \rightarrow \mathscr{C}_{M I}\right|_{I}
$$

In virtue of proposition 1.4 the structure of $\mathscr{C}_{M+/ X}$ is understood except for on $I$.

Proposition 1.5. The following sequence is exact on $S_{W^{+}}^{*} X$ $-S_{Y} * X ; \quad \pi_{M_{+} / X}^{-1} \Gamma_{M_{+}} \mathcal{B}_{M} \rightarrow \mathscr{C}_{M_{+} / X} / \mathscr{C}_{N / X} \rightarrow 0$

In order to treat these sheaves more explicitly, recall the following result which was obtained by Kashiwara-Kawai.

Proposition 1.6. (Kashiwara-kawai [5], [6]). Let $U_{k}^{ \pm}=\left\{\left(0, x^{\prime}\right.\right.$; $\left.\left.\zeta_{1}, i \eta^{\prime}\right) \in T_{N}{ }^{*} X ; \eta_{k}= \pm 1\right\} \subset S_{N}{ }^{X} X-S_{Y}{ }^{*} X$ be open subset of $S_{N}{ }^{*} X$ for every $k, 2 \leqq k \leqq n$. Then on $U_{k}^{ \pm}$we have the following isomorphisms;

$$
b_{k}{ }^{ \pm} ;\left.\mathscr{C}_{N / X i} U_{k^{ \pm}} \rightarrow \mathscr{C O O}\right|_{U_{k^{ \pm}}}
$$

Here, $\mathscr{C} O$ is the sheaf of microfunctions of $\left(\zeta_{1}, x^{\prime}\right)$ with holomorphic parameter $\zeta_{1} . \quad\left(U_{k}^{ \pm} \subset \mathbb{C} \times i S^{*} \mathbb{R}^{n-1} \ni\left(\zeta_{1}, x^{\prime} ; i \eta^{\prime}\right)\right)$

Definition 1.7. We denote by $\gamma^{+}, \gamma$, the following natural mappings; $\quad \gamma^{+} ; \bar{G}_{+}-S_{Y}{ }^{*} X \rightarrow S_{N}{ }^{*} Y\left(=i S^{*} N\right)$

$$
r ; S_{N}^{*} X-S_{Y}^{*} X \rightarrow i S^{*} N
$$

As a direct corollary of Proposition 1.6, we obtain the unique continuation property of $\mathscr{C}_{N / X}$ along the fiber of $\gamma$. Further we can extend this fact to the case of $\mathscr{C}_{M_{+} / X}$. (the equivalent result has already been obtained by M. Kashiwara. [4], [1], [12]) 
Proposition 1.8. $\left.\Gamma_{\bar{H}_{+}} \mathscr{C}_{M_{+} / X}\right|_{I}=0$. Considering the fact, " $\left.\mathscr{C}_{M_{+} / X}\right|_{G_{+}}$ $=\left.\mathscr{C}_{N / X}\right|_{G_{+}} "$, this means the unique continuation property of $\mathscr{C}_{M_{+} / X}$ along the fiber of $\gamma^{+}$.

By this fact we can identify the germ of $\mathscr{C}_{M_{+} / X}$ on $I$ with a section of $\left.\mathscr{C}_{N / X}\right|_{G_{+}}$, that is, the following homomorphism is canonically induced and injective;

$$
\Phi ;\left.\left.\mathscr{C}_{M_{+} / X}\right|_{1} \rightarrow t_{*}\left(\left.\mathscr{C} O\right|_{G_{+}}\right)\right|_{I}
$$

Here, $t$ is the imbedding mapping from $G_{+}$to $\bar{G}_{+}$. Moreover by Proposition 1.6 we obtain the following injective homomorphism.

$$
b_{k}^{ \pm} \cdot \Phi ;\left.\left.\mathscr{C}_{M^{+} / X}\right|_{I \cap U_{k^{ \pm}}} \rightarrow t_{*}\left(\left.\mathscr{C} O\right|_{G^{+}}\right)\right|_{I \cap U_{k^{*}}}
$$

Then concerning $\operatorname{Im}\left(b_{k}{ }^{ \pm} \cdot \Phi\right)$, we have;

Proposition 1.9. We set $L=\mathbb{C} \times \boldsymbol{R}^{n-1} \ni\left(\zeta_{1}, x^{\prime}\right), L_{+}=\left\{\left(\zeta_{1}, x^{\prime}\right) \in L\right.$; $\left.\operatorname{Re} \zeta_{1}>0\right\}$. We denote by $\tau$ the imbedding mapping from $L_{+}$to $L$, and by $\mathcal{B O}$ the sheaf of hyperfunctions defined on $L$ with holomorphic parameter $\zeta_{1}$. Then the germ of $\left.t_{*}\left(\left.\mathscr{C} O\right|_{G+}\right)\right|_{I \cap U_{k^{*}}}$ belongs to $\operatorname{Im}\left(b_{k}{ }^{ \pm} \cdot \Phi\right)$ if and only if it is represented by the germ of $\left.\pi^{-1} \tau_{*}\left(\left.\mathscr{B O}\right|_{L_{+}}\right)\right|_{I_{\cap U_{k}} \text {. }}$ (here, $\pi ; \boldsymbol{C} \times i S^{*} \mathbb{R}^{n-1} \rightarrow \mathbb{C} \times \mathbb{R}^{n-1}=L$ )

\section{$\S 2$. Several Operations}

We denote by $\mathscr{P}_{X}$ the sheaf of micro-differential operators (S-K-K [10]). This sheaf is defined on $S^{*} X$, and especially on $S_{M^{+}}^{*} X$ or $S_{N}^{*} X$.

Proposition 2.1. $\mathscr{C}_{M_{+} / X}$ and $\mathscr{C}_{N / X}$ are canonically considered as $\mathscr{L}_{X}$-modules. Moreover, let $\Psi ; T^{*} X \supset U \rightarrow V \subset T^{*} X$ be a complex contact transformation which keeps $T_{M_{+}}^{*} X$ (or $T_{N}{ }^{*} X$ ), then the quantized constact transformation attached to $\Psi$ induces the following sheaf isomorphism;

$$
\begin{aligned}
& \left.\left.\mathscr{C}_{M_{+} / X}\right|_{U \cap S_{N+}^{*} X} \rightarrow \mathscr{C}_{M+/ X}\right|_{V \cap S_{N+}^{*} X} \\
& \left(\left.\left.\mathscr{C}_{N / X}\right|_{U \cap S_{N}^{*} X} \rightarrow \mathscr{C}_{N / X}\right|_{V \cap S_{N}^{*} X}\right)
\end{aligned}
$$

Remark. For a complex contact transformation $\Psi$, the following 
statements are all equivalent.
A. $\Psi$ keeps $T_{M I^{+}}^{*} X$.
B. $\Psi$ keeps $T_{N}{ }^{*} X$ and $i T^{*} M$ respectively.
C. $\Psi$ keeps $\pi^{-1}(Y)$ and $i T^{*} M$ respectively.

Proposition 2.2. Let $p^{\prime}$ be any point on $i S^{*} N, K$ be any compact subset of $\gamma^{-1}\left(p^{\prime}\right)$ such that the boundary of $K$ is a real analytic Jordan curve, and $P(x, D)$ be a micro differential operator defined on the neighborhood of $\gamma^{-1}\left(p^{\prime}\right)$ such that; $P(x, D)=D_{1}{ }^{m}+P_{1}\left(x, D^{\prime}\right)$ $\times D_{1}{ }^{m-1}+\cdots+P_{m}\left(x, D^{\prime}\right)$, where for every $j(1 \leqq j \leqq m),\left[P_{j}\left(x, D^{\prime}\right), x_{1}\right]$ $=0$ and ord. $P_{j} \leqq j$. Moreover we suppose $\sigma_{m}(p) \neq 0$ on $\gamma^{-1}\left(p^{\prime}\right)-K$, then it follows that; $\Gamma\left(K, \mathscr{C}_{N / X}\right)=\left.P(x, D) \Gamma\left(K, \mathscr{C}_{N / X}\right) \bigoplus_{j=0}^{m-1} \mathscr{C}_{N}\right|_{p^{\prime}} \cdot \delta^{(j)}\left(x_{1}\right)$ And the projection operators of this direct decomposition is a kind of micro-differential operator whose symbols are explicitly calculated by the symbols of $P(x, D)$ in turn from higher order to lower order.

\section{§ 3. Micro Localication of Non-Characteristic Boundary Value Problems}

Let $P(x, D)$ be a differential operator of order $m$ defined on $M$ with respect to which $N$ is non-characteristic. Then non-characteristic boundary value problems will be reduced to the study of interdependence between solutions in $\Gamma_{M+} \mathcal{B}_{M} / \Gamma_{N} \mathcal{B}_{M}$ of $P u^{\prime}=0$, and their boundary values. That is, corresponding to every solutions of $P u^{\prime}=0$, the pair of a germ of $\left.\Gamma_{M_{+}} \mathcal{B}_{M}\right|_{N}{ }^{\prime \prime} u(x)$ " and a germ of $\mathcal{B}_{N}{ }^{m}$ " $\left(f_{0}\left(x^{\prime}\right), \cdots, f_{m-1}\left(x^{\prime}\right)\right)$ " exists uniquely under the following conditions;

$$
P(x, D) u(x)=\sum_{j=0}^{m-1} f_{j}\left(x^{\prime}\right) \delta^{(j)}\left(x_{1}\right), u^{\prime}(x)=[u(x)] .
$$

And we call $\left(f_{0}, \cdots, f_{m-1}\right)$ boundary values of $u^{\prime}$ with respect to $P$.

Now we return to the original situation of $\S \mathbb{1}$ and $\S \mathbf{2}$, then by Proposition $1.3 u(x)$ and $F(x)=\sum_{j=1}^{m-1} f_{j}\left(x^{\prime}\right) \delta^{(j)}\left(x_{1}\right)$ are identified with sections of $\mathscr{C}_{M^{+} / X}$ and $\mathscr{C}_{N / X}$ respectively, and the equation " $P u=F$ " is regarded as an equation defined on $\bar{G}_{+}$.

Moreover it is important that the solution " $u(x)$ " of $P u=F$ is micro locally unique at any point on $\bar{G}_{+}$if it exists. 
Consequently the study will be reduced to the study in the sense of micro local theory. Hence for the study of this problem we can use the standard methods, "Weierstrass'es division theorem for $\Psi$.D.O.", or "quantized contact transformations which keep $S_{M_{+}}^{*} X$ ", though by these operations the property of $P$ as a differential operator is lost. So it will be convenient to formulate non-characteristic boundary value problems in the sense of micro local theory.

Definition 3.1. We call $P(x, D) \in \gamma^{*} \mathscr{L}_{X}^{f}$, non-characteristic microdifferential operator with respect to $N$, if $\{\sigma(P)=0\} \cap\left(S_{N}{ }^{*} X-S_{Y}{ }^{*} X\right)$ $\stackrel{r}{\rightarrow} i S^{*} N$ is a proper mapping. Clearly the fiber of this mapping is a finite set, and the number of this set including the multiplicity is locally constant. Then we call this number the essential order of $P$.

Remark. By Weierstrass' division theorem for $\Psi$.D.O., the abovementioned $P$ is decomposed in the following way; $P=P_{1} \cdot P_{2}$, where, $P_{1}$, $P_{2} \in \gamma^{*} \mathcal{L}_{X}^{f}, P_{2}$ is elliptic, and

$$
P_{1}(x, D)=D_{1}{ }^{m}+Q_{1}\left(x, D^{\prime}\right) D_{1}{ }^{m-1}+\cdots+Q_{m}\left(x, D^{\prime}\right)
$$

(ord. $Q_{j} \leqq j$, for any $j$, and $m$ is the essential order of $P$ )

Let $P(x, D)$ be a non-characteristic micro-differential operator, then taking account of Proposition 2.2, we can formulate the boundary value problem for $P$ as the study of interdependence between $\gamma_{*}{ }^{+} \mathscr{C}_{M_{+} / X} / \gamma_{*} \mathscr{C}_{N / X^{-}}$ solutions of $P$, and their boundary values.

In virtue of Proposition 2.2, the analysis of this problem on $G_{+}$is easy, and the following result is obtained.

Theorem 3.2. ${ }^{\forall} p_{0}{ }^{\prime} \in i S^{*} N,\left.P(x, D) \in \gamma_{*} \mathcal{L}_{X}{ }^{f}\right|_{p_{0}}$, ess. order of $P=m$. Then for $\left.\left(f_{0} \cdots f_{m-1}\right) \in \mathscr{C}_{N}{ }^{m}\right|_{p_{0}{ }^{\prime}}$, the equation

$$
P(x, D) u(x)=\sum_{j=0}^{m-1} f_{j}\left(x^{\prime}\right) \delta^{(j)}\left(x_{1}\right)
$$

is solvable on $G_{+} \cap \gamma^{-1}\left(p_{0}{ }^{\prime}\right)$ if and only if the following equations are satisfied;

$$
f_{k}\left(x^{\prime}\right)=\sum_{j=s}^{m-1} Q_{k j}\left(x^{\prime}, D^{\prime}\right) f_{j}\left(x^{\prime}\right), \text { for } O \leqq \forall k \leqq s-1 \text {, at } p_{0}^{\prime} . \quad \text { Here, } s
$$


$=\frac{\psi}{\psi}\left(\{\sigma(P)=0\} \cap G_{\perp} \cap \gamma^{-1}\left(p_{0}{ }^{\prime}\right)\right)$, (including the multiplicity) and $Q_{k j}$ $\left.\left(x^{\prime}, D^{\prime}\right) \in \mathscr{L}_{Y}^{f}\right|_{p_{0^{\prime}}} \quad(0 \leqq \forall k \leqq s-1, s \leqq \forall j \leqq m-1)$, whose symbols are calculated by the symbols of $P$.

\section{References}

[1] Bony, J. M., Extensions du theoreme de Holmgren, Séminaire Goulaouic-Schwartz 1975-1976.

[2] Kaneko, A., Singular Spectrum of Boundary Values of Solutions of Partial Differential Equations with Real Analytic Coefficients, Sci. Pap. Coll. Gen. Educ. Univ. Tokyo 25 (1975), 59-68.

[3] Kashiwara, M., Algebraic foundation of the theory of hyperfunctions, Surikaisekikenkyusho Kokyuroku No. 108, R.I.M.S. Kyoto Univ., 1969. (Japanese)

[4] Kashiwara, M., Exposé oral au colloque "Hyperfonctions et Physique Théorique" (1972) Nice (non publié).

[5] Kashiwara, M., Kawai, T., On the boundary value problem for elliptic system of linear differential equations, I. Proc. Japan Acad., 48 (1972).

[6] - II, Proc. Japan Acad., 49 (1973).

[7] Kataoka, K., On the theory of Radon transforms for hyperfunctions and its applications, Master's thesis, Univ. Tokyo 1976 (Japanese).

[8] Komatsu, H., Kawai, T., Boundary values of hyperfunction solutions of linear differential equations, Publ. Res. Inst. Math. Sci., 7 (1971).

[9] Morimoto, M., Sur les ultradistributions cohomologiques, Ann. Inst. Fourier, 19-2 (1969).

[10] Sato, M., Kawai, T., Kashiwara, M., Microfunctions and pseudo-differential equations, Lecture Notes in Math. No. 287, Springer (1973).

[11] Schapira, P., Hyperfonctions et problèmes aux limites elliptiques, Bull. Soc. Mat. France 99, (1971), 113-141.

[12] Schapira, P., Propagation au bord et reflexion des singularites analytiques des solutions des equations aux derivees partielles, Seminaire Goulaouic-Schwartz 19751976. 
